\title{
Histone modifications around individual BDNF gene promoters in prefrontal cortex are associated with extinction of conditioned fear
}

\author{
Timothy W. Bredy, ${ }^{1}$ Hao Wu, ${ }^{1}$ Cortney Crego, ${ }^{1}$ Jessica Zellhoefer, ${ }^{1}$ Yi E. Sun, ${ }^{1}$ \\ and Mark Barad ${ }^{1,2,3}$ \\ ${ }^{1}$ Department of Psychiatry and Biobehavioral Sciences, Brain Research Institute, Semel Institute for Neuroscience and Human \\ Behavior, Los Angeles, California 90095, USA; ${ }^{2}$ West Los Angeles VA Medical Center, Los Angeles, California 90073, USA
}

\begin{abstract}
Extinction of conditioned fear is an important model both of inhibitory learning and of behavior therapy for human anxiety disorders. Like other forms of learning, extinction learning is long-lasting and depends on regulated gene expression. Epigenetic mechanisms make an important contribution to persistent changes in gene expression; therefore, in these studies, we have investigated whether epigenetic regulation of gene expression contributes to fear extinction. Since brain-derived neurotrophic factor (BDNF) is crucial for synaptic plasticity and for the maintenance of long-term memory, we examined histone modifications around two BDNF gene promoters after extinction of cued fear, as potential targets of learning-induced epigenetic regulation of gene expression. Valproic acid (VPA), used for some time as an anticonvulsant and a mood stabilizer, modulates the expression of BDNF, and is a histone deacetylase (HDAC) inhibitor. Here, we report that extinction of conditioned fear is accompanied by a significant increase in histone $\mathrm{H} 4$ acetylation around the BDNF P4 gene promoter and increases in BDNF exon I and IV mRNA expression in prefrontal cortex, that VPA enhances long-term memory for extinction because of its HDAC inhibitor effects, and that VPA potentiates the effect of weak extinction training on histone $\mathrm{H} 4$ acetylation around both the BDNF P1 and P4 gene promoters and on BDNF exon IV mRNA expression. These results suggest a relationship between histone $\mathrm{H} 4$ modification, epigenetic regulation of BDNF gene expression, and long-term memory for extinction of conditioned fear. In addition, they suggest that HDAC inhibitors may become a useful pharmacological adjunct to psychotherapy for human anxiety disorders.
\end{abstract}

Substantial evidence indicates that extinction of conditioned fear, the reduction in responding to a feared cue when the cue is repeatedly presented without any adverse consequence, is new learning that inhibits the expression of a conditioned association rather than erasing it. For example, conditioned fear shows "spontaneous recovery" after the passage of time (Baum 1988), "reinstatement" after presentations of the unconditioned stimulus (US) alone (Rescorla and Heth 1975), and "renewal" when the feared cue is presented in a context different from that of extinction training (Bouton and King 1983). Efforts to understand the mechanisms of this form of learning have increased recently, particularly since it is an important model of anxiety disorder treatment.

Many forms of learning, including extinction, are dependent on changes in gene expression (Berman and Dudai 2001; Cammarota et al. 2003; Lin et al. 2003; Sangha et al. 2003; Vianna et al. 2003; Herry and Mons 2004; Suzuki et al. 2004; Yang and Lu 2005; Chhatwal et al. 2006; Herry et al. 2006; Lattal et al. 2006). Dynamic changes in chromatin structure make an important contribution to the regulation of tissue-specific gene expression. In particular, histone acetylation/deacetylation and dimethylation of specific lysine residues on nucleosomal histone proteins (i.e., H3-K9) and DNA methylation of CpG dinucleotides within promoter regions are ways that chromatin remodeling can influence ongoing transcription and synaptic plasticity (Martinowich et al. 2003; Levenson et al. 2006). Histone acety-

\footnotetext{
${ }^{3}$ Corresponding author.
}

E-mail mbarad@mednet.ucla.edu; fax (310) 825-9870.

Article is online at http://www.learnmem.org/cgi/doi/10.1101//m.500907. lation contributes an early step to the process of chromatin modification by disassembling nucleosomes to make DNA promoter regions accessible for transcription factor binding and for methylation. Histone acetylation states are regulated by specific enzymes, including histone deacetylases (HDACs), which can be both tissue- and cell-type-specific. Thus, the omnipresence and specificities of these enzymes may make them potential therapeutic targets for the treatment of neuropsychiatric disorders and disorders of learning and memory.

In addition to its trophic function during development, brain-derived neurotrophic factor (BDNF) is critical for learningrelated synaptic plasticity and the maintenance of long-term memory. The role of BDNF in fear conditioning is well defined, and, within the amygdala of the rat, both fear conditioning and its extinction lead to an increase in BDNF protein and gene transcripts (Rattiner et al. 2004; Chhatwal et al. 2006; Ou and Gean 2006). Recent data indicate that the medial prefrontal cortex also plays an important role in fear extinction learning (Milad and Quirk 2002; Milad et al. 2004; Santini et al. 2004), but the function of BDNF in the prefrontal cortex during extinction remains undefined. Thus, regulation of BDNF in the prefrontal cortex is a reasonable candidate mechanism to make a contribution to extinction learning.

BDNF has four distinct transcripts each regulated by a specific promoter that is sensitive to epigenetic modification (Martinowich et al. 2003; Tsankova et al. 2004). We chose to examine histone acetylation around two of those promoters in the prefrontal cortex after fear conditioning or after fear conditioning followed by fear extinction in order to provide support for the hypothesis that distinct patterns of histone acetylation are asso- 
ciated with specific behavioral changes: histone acetylation patterns and behavior that might be mimicked by combining partial behavioral training with a drug that promotes histone acetylation.

We find that fear conditioning and extinction result in distinct patterns of histone acetylation of histones $\mathrm{H} 3$ and $\mathrm{H} 4$ around the P1 and P4 promoters of the BDNF gene. We also find that promoting histone acetylation potentiates partial fear extinction, by decreasing remembered fear, but only when combined with partial extinction training (training insufficient to generate significant extinction on its own). Finally, we demonstrate that the same partial extinction training, by itself, also fails to generate the characteristic histone acetylation changes of extinction, but, when combined with an HDAC inhibitor, mimics the histone acetylation pattern characteristic of strong extinction around the BDNF $\mathrm{P} 4$ promoter. This synergistic combination also increases BDNF exon IV-containing transcripts, while the combination does not specifically affect exon I-containing transcripts.

\section{Results}

\section{Extinction of conditioned fear} results in histone acetylation around BDNF gene promoters within the prefrontal cortex Behavioral training resulted in significant changes in $\mathrm{H} 3$ acetylation around the $\mathrm{P} 1$ promoter of the BDNF gene (oneway ANOVA, $F_{(2,8)}=58.70, P<0.0001$; Fig. 1A). Fear-conditioned mice without extinction showed a significant increase in $\mathrm{H} 3$ acetylation compared to naive controls (Bonferroni post hoc analysis; $P<0.01$ ), suggesting an increase in histone acetylation due to fear conditioning. Extinction training reversed this $\mathrm{H} 3$ acetylation to below naive levels $(P<0.01$ vs. fear conditioned without extinction; $P<0.05$ vs. naive). There was no significant effect of learning on $\mathrm{H} 4$ acetylation around the $\mathrm{P} 1$ promoter of the BDNF gene (Fig. 1B).

Behavioral training led to an increase in acetylated $\mathrm{H} 3$ around the $\mathrm{P} 4$ promoter of the BDNF gene (one-way ANOVA, $F_{(2,8)}=5.92, P<0.05$; Fig. 1D). Fear-conditioned without extinction mice showed a significant increase of $\mathrm{H} 3$ acetylation at this promoter compared to naive animals $(P<0.05)$, while increases in extinguished animals were not quite significant. There was no difference in $\mathrm{H} 3$ acetylation between fear-conditioned mice without extinction and extinguished animals $(P>0.05)$. Training also affected acetylated $\mathrm{H} 4$ at this promoter $\left(F_{(2,8)}=15.13, P<0.01\right.$; Fig. 1E). Specifically, although fear conditioning without extinction caused no change in histone acetylation compared to naive animals $(P>0.05)$, extinction training increased $\mathrm{H} 4$ acetylation around the $\mathrm{P} 4$ promoter of the BDNF gene relative to both other groups $(P<0.01)$. There was no significant learning-induced histone modification around the control promoter for $\beta$-tubulin (Fig. 1G-I).
Extinction of conditioned fear results in increased BDNF mRNA expression within the prefrontal cortex

Behavioral training resulted in a significant increase in BDNF exon I mRNA expression (one-way ANOVA, $F_{(2,9)}=31.32$, $P<0.0001$; Fig. 2B). Fear-conditioned mice without extinction showed no increase in BDNF exon I mRNA compared to naive controls; however, extinction training increased BDNF exon I mRNA expression relative to both naive and fear-conditioned without extinction mice (Bonferroni post hoc tests, $P<0.01$ ) (Fig. 2B).

Behavioral training resulted in a significant increase in BDNF exon IV mRNA expression (one-way ANOVA, $F_{(2,9)}=22.85, P<0.001$; Fig. 2D). Fear-conditioned mice without extinction showed no increase in BDNF exon IV mRNA compared to naive controls; however, extinction training increased BDNF exon IV mRNA expression relative to both naive and fearconditioned without extinction mice (Bonferroni post hoc tests, $P<0.01)$ (Fig. 2D).

Histone deactylase inhibitors, given prior to training, enhance long-term memory for the extinction of conditioned fear

Valproic acid (VPA) has been used for decades as an anticonvulsant and a mood stabilizer (Winterer and Hermann 2000); how- 
A

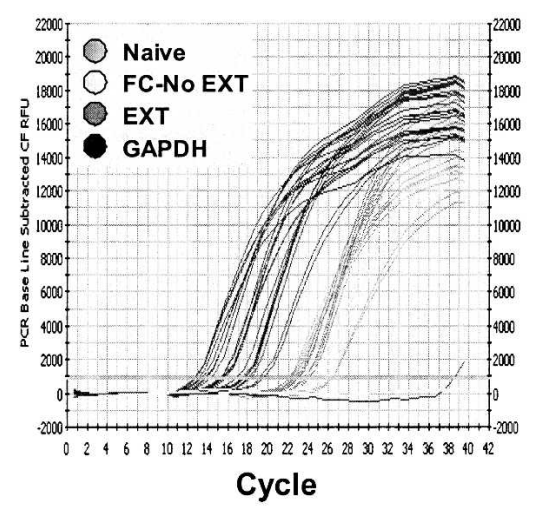

C

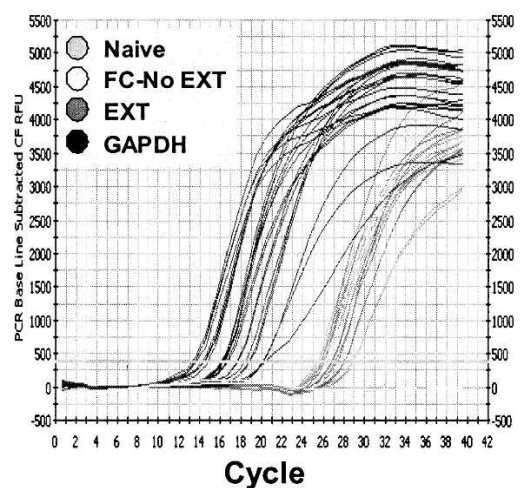

B

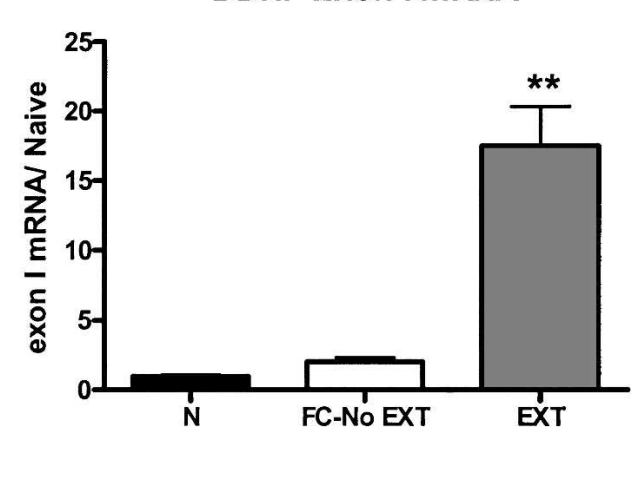

D

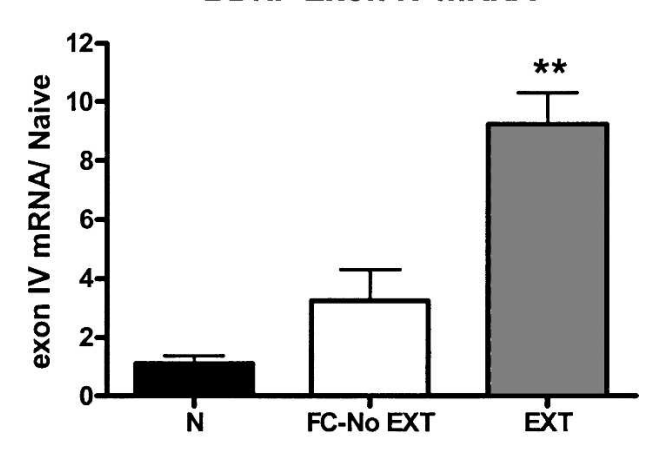

Figure 2. Effect of learning on $(A, B)$ BDNF exon I and $(C, D)$ IV mRNA expression in the prefrontal cortex. $(A)$ Representative quantitative RT-PCR plot for the effect of strong (57 CSs) extinction training on BDNF exon I mRNA expression in the prefrontal cortex. (B) Strong extinction training yielded a significant increase in BDNF exon I mRNA expression. (C) Representative quantitative RT-PCR plot for the effect of strong (57 CSs) extinction training on BDNF exon IV mRNA expression in the prefrontal cortex. $(D)$ Strong extinction training yielded a significant increase in BDNF exon IV mRNA expression. $\left({ }^{* *} P<0.01, n=4\right.$ samples pooled from two animals each.) (N) Naive; (FC-No EXT) fear conditioned without extinction; (EXT) partial extinction.

ever, it is also a potent HDAC inhibitor (Phiel et al. 2001) and increases BDNF mRNA and protein levels both in cell culture (Castro et al. 2005) and in the prefrontal cortex (Fukumoto et al. 2001). When administered chronically, VPA reduces fear responding after nonreinforced extinction-like exposures to an aversive context (Li et al. 2006), and a derivative of VPA, pentyl4-yn-valproic acid, enhances both spatial learning and long-term memory for avoidance conditioning (Murphy et al. 2001; Foley et al. 2004). We therefore injected VPA before a partial extinction protocol as previously described (Cain et al. 2003; Ponnusamy et al. 2005). By itself, this protocol yields no significant long-term memory for extinction.

VPA had no effect on long-term memory (LTM) for fear of the CS (measured by behavioral freezing) when injected on day 2 in the absence of CS presentations (see FC-No EXT groups, Fig. $3 \mathrm{~A})$ nor on the rate of within-session extinction across $20 \mathrm{CS}$ exposures (data not shown), but enhanced long-term memory for extinction in a dose-dependent, U-shaped manner $(n=16 /$ group, $F_{(5,94)}=6.91, P<0.0001$; Bonferroni post hoc analysis, $P<0.05$ vehicle vs. $100 \mathrm{mg} / \mathrm{kg}$ ). The U-shape may have resulted from the sedating effects of the highest dose preventing learning during extinction training. There was a main effect of VPA treatment on pre-CS freezing after partial extinction training $\left(F_{(5,94)}=2.92, P<0.05\right.$; Fig. 3A, left $)$; however, when pairwise comparisons were made, Bonferroni post hoc analysis revealed no significant difference in freezing between vehicletreated and VPA-treated mice In contrast, valpromide, an analog of VPA that shares its anticonvulsant and moodstabilizing effects but is not an HDAC inhibitor (see chemical structures in Fig. $4)$, had no effect on LTM for extinction $\left(n=8-16 /\right.$ group, $F_{(5,66)}=0.9078$, $P=0.48$; Fig. 3B). Conversely, sodium butyrate $(\mathrm{NaBt})$, a prototypical HDAC inhibitor with no known anticonvulsant activity, also increased LTM for fear extinction $\left(n=15-16 /\right.$ group, $F_{(5,95)}=5.80$, $P<0.0001, P<0.05$, vehicle vs. 1000 $\mathrm{mg} / \mathrm{kg}$ ) (Fig. 3C). There was no effect of sodium butyrate treatment on pre-CS freezing scores (Fig. 3C, left).

\section{VPA, combined with partial extinction training, increased histone $\mathrm{H} 4$ acetylation around both the BDNF Pl and P4 gene promoters but increased only BDNF exon IV mRNA expression in prefrontal cortex}

To provide evidence for a more direct relationship between changes in histone acetylation in the prefrontal cortex and extinction learning, we tested the effect of VPA $(100 \mathrm{mg} / \mathrm{kg})$ combined with partial extinction training on histone modifications around the BDNF P1 and P4 promoters as well as on BDNF exon I and IV transcripts using the same extinction protocol used above to analyze the effect of HDAC inhibitors on behavior.

There was no significant effect of drug treatment or training on H3 acetylation at the BDNF P1 promoter $(n=4$ samples pooled from two animals each, two-way ANOVA, $F_{(1,12)}=2.71$, ns; Fig. 5A). On the other hand, VPA treatment caused a significant increase in $\mathrm{H} 4$ acetylation overall at this promoter (two-way ANOVA, $F_{(1,12)}=3.48, P<0.05$ for treatment) with a trend toward interaction between training and drug treatment $(P=0.076)$ (Fig. 5B). Partial extinction training alone yielded no significant increase in $\mathrm{H} 4$ acetylation, consistent with the absence of an effect on freezing. However, this VPA-induced increase in $\mathrm{H} 4$ acetylation was unrelated to BDNF exon I mRNA expression (Fig. 5D). While there was a significant main effect of partial extinction training on BDNF exon I mRNA expression $(n=6$ samples, pooled from two animals each; $\left.F_{(1,20)}=6.20, P<0.05\right)$, there was neither interaction nor main effect of VPA on the expression of BDNF exon I.

Neither partial extinction training nor VPA affected H3 acetylation at the $\mathrm{P} 4$ promoter $(n=4$ samples pooled from two animals each, 2 -way ANOVA, $F_{(1,12)}=1.563$, ns; Fig. 6 A). However, there was a significant interaction between training and drug treatment for $\mathrm{H} 4$ acetylation there (two-way ANOVA, $F_{(1,12)}=10.66, P<0.02$; Fig. 6B). Partial extinction training yielded no significant increase in $\mathrm{H} 4$ acetylation, in parallel with its lack of effect on freezing. VPA alone also had no effect on H4 acetylation. However, VPA significantly increased $\mathrm{H} 4$ acetylation 
in combination with partial extinction training $(P<0.01)$, again paralleling its behavioral effect in facilitating extinction (Fig. 6A). This increase in $\mathrm{H} 4$ acetylation by the combination of VPA and partial extinction training also mimicked the effect of strong extinction training alone. Unlike the result for BDNF exon I mRNA, there was a significant interaction between training and drug treatment for exon IV mRNA (two-way ANOVA, $F_{(1,20)}=11.97$, $P<0.01, n=6$ samples, pooled from two animals each; Fig. 6D). Bonferroni post hoc tests indicate that partial extinction training only increased exon IV mRNA when combined with VPA $(P<0.01)$. Thus, even though VPA may inhibit HDACs nonspecifically, its effects on histone acetylation at the P4 BDNF promoter and on gene expression of BDNF exon IV in the prefrontal cortex are quite specific to the pairing of VPA with behavioral experience.

A

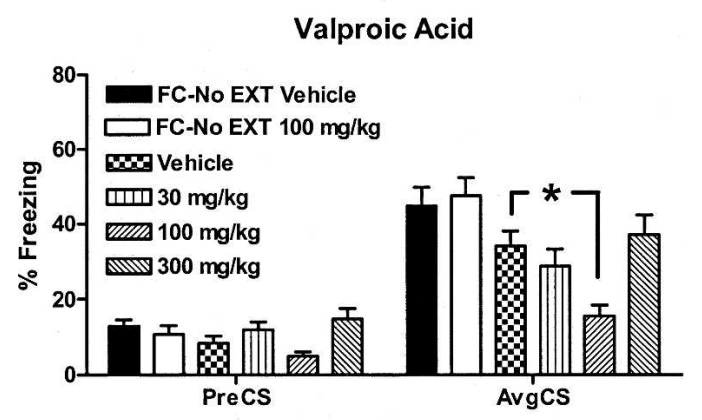

B

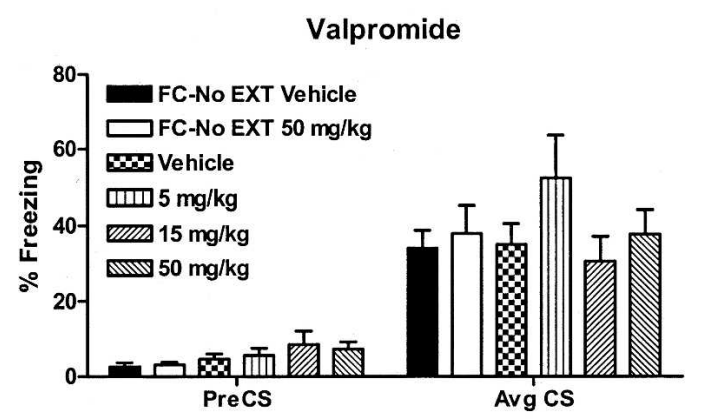

C

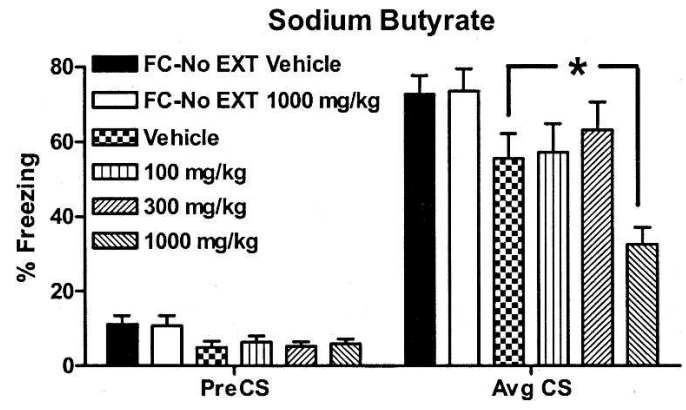

Figure 3. Mean \pm SEM percent freezing $7 \mathrm{~d}$ after histone deacetylase (HDAC) inhibitor injection before partial extinction training. None of the drugs had any significant effect on pre-CS freezing compared to mice treated with vehicle and partially extinguished. $(A)$ At a dose of 100 $\mathrm{mg} / \mathrm{kg}$, valproic acid potentiates long-term memory for the extinction of conditioned fear. (B) Valpromide has no effect on extinction of conditioned fear. (C) At a dose of $1000 \mathrm{mg} / \mathrm{kg}$, sodium butyrate potentiates long-term memory for the extinction of conditioned fear. $\left({ }^{*} P<0.05\right.$ compared to mice extinguished with vehicle; $n=8-16$ mice/group.) (FCNo EXT) Fear conditioned without extinction.
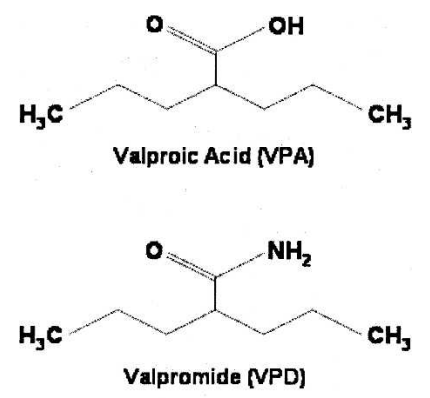<smiles>CCCC(=O)O</smiles>

Sodium Butyrate (NaBt)

Figure 4. Chemical structures of compounds used to facilitate extinction.

\section{Discussion}

This study generated four main findings: (1) Acquisition and extinction of conditioned fear results in distinct histone modifications around two BDNF gene promoters in the prefrontal cortex. (2) Extinction of conditioned fear leads to an increase in BDNF mRNA expression in the prefrontal cortex. (3) Histone deacetylase inhibitors, including VPA, given prior to partial extinction training, enhance long-term memory for the extinction of conditioned fear. (4) VPA, given prior to partial extinction training, increases histone $\mathrm{H} 4$ acetylation at both the BNDF P1 and P4 gene promoters, reproducing the acetylation pattern of strong extinction on $\mathrm{P} 4$, but the combination only increases BDNF exon IV-containing mRNA expression in the prefrontal cortex.

The increase in $\mathrm{H} 4$ acetylation around the $\mathrm{P} 4$ promoter after extinction (Fig. 1E) is consistent with a previous report that $\mathrm{H} 4$ acetylation is generally increased in the hippocampus after latent inhibition of conditioned fear, another inhibitory learning protocol (Levenson et al. 2004). However, the change we see in H4 acetylation is promoter-specific, even in prefrontal cortex. H4 acetylation is significantly increased around the $\mathrm{P} 4$ promoter, but only shows a trend to increase around the P1 promoter (Fig. 1B) after extinction training. Also, we have seen no such H4 acetylation increases after extinction in either the hippocampus or amygdala at these BDNF promoters (data not shown). Conversely, while $\mathrm{H} 3$ acetylation is increased around both promoters in the prefrontal cortex by fear conditioning without extinction (Fig. 1A,D), extinction training has opposite effects on H3 acetylation at the two promoters. Thus, our data support the hypothesis that there potentially exists a histone code for different forms of learning and memory (Chwang et al. 2006; Wood et al. 2006), since we show specific histone modifications around individual promoters of the BDNF gene in response to specific learning protocols.

While the contrast in histone acetylation between fearconditioned without extinction and naive animals is striking for $\mathrm{H} 3$, it is important to be cautious about the interpretation of these results, since naive animals were exposed to neither the conditioning context, the CS, the US, nor the second context used for extinction. Thus, any of these factors may be influencing the changes in histone acetylation we see in fear conditioning without extinction compared to naive animals in a nonspecific manner regardless of fear conditioning. However, the contrast in histone acetylation between fear-conditioned without extinction and extinguished mice (or, for that matter, the lack of contrast for $\mathrm{H} 3$ at P4 and for $\mathrm{H} 4$ at P1) cannot depend on such nonspecific 


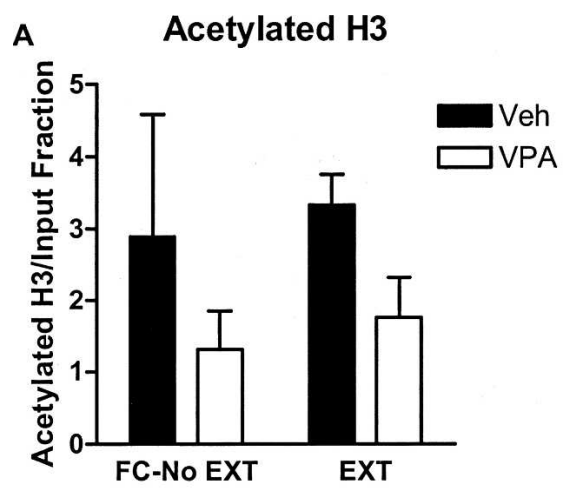

C

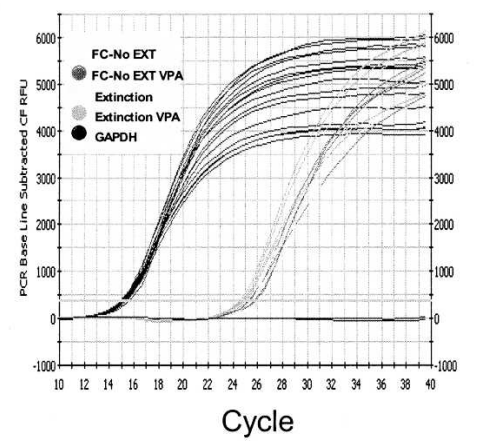

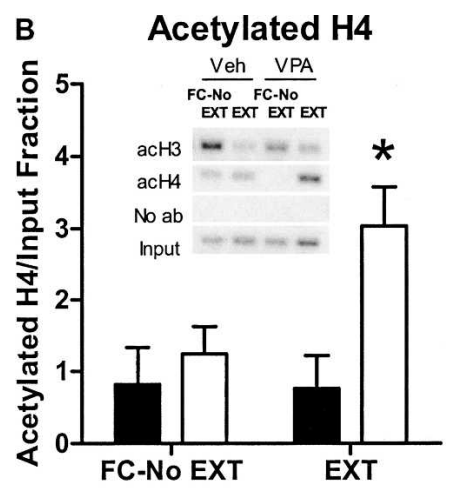

D BDNF Exon I mRNA

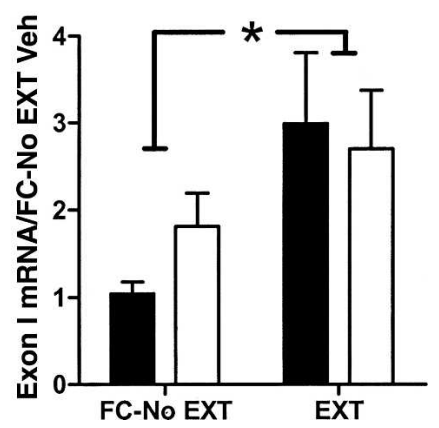

Figure 5. Effect of histone deacetylase inhibition by valproic acid (VPA) on learning-induced histone modifications ( \pm SEM) around the P1 promoter of the BDNF gene and on BDNF exon I mRNA expression in the prefrontal cortex. VPA $(100 \mathrm{mg} / \mathrm{kg})$ combined with partial extinction training (20 CS presentations) $(A)$ had no significant effect on $\mathrm{H} 3$ acetylation around the $\mathrm{P} 1$ promoter, $(B)$ but increased histone $\mathrm{H} 4$ acetylation relative to all other treatment groups $\left({ }^{*} P<0.05, n=4\right.$ samples pooled from two animals each). (Inset) Raw data. (C) Representative quantitative RT-PCR plot for the effect of VPA and partial extinction training on BDNF exon I mRNA expression in the prefrontal cortex. (D) Extinction training yielded a significant increase in BDNF exon I mRNA expression. ( ${ }^{*} P<0.05, n=6$ samples pooled from two animals each.) (FC-No EXT) Fear conditioned without extinction; (EXT) partial extinction.

factors, since both groups received exactly the same training in context A on the first day, and then spent the same amount of time in context $\mathrm{B}$ on the second day. The only difference between these groups is the CS presentations on day 2. So while it remains possible that repeated CS exposures alone generate changes in histone acetylation (regardless of previous training), we believe that these changes are most likely to be due to extinction learning. This is supported by the correlation of such changes with extinction learning and not with CS exposures in the extinction paradigm used in our later experiments (Figs. 4-6). No change in histone acetylation was observed in groups that received only extinction training and vehicle, despite the presentation of a substantial number of CSs to those animals. In fact, histone $\mathrm{H} 4$ acetylation only increased around the P1 and P4 promoters under conditions that yielded effective extinction learning after the injection of VPA in combination with partial extinction training (Figs. 4-6).

Interestingly, when given before extinction training, VPA induced reductions in pre-CS freezing similar to those in freezing to the CS (Fig. 4A). Although not significant, these data suggest the possibility that VPA may influence extinction through contextual rather than cue-based learning. Nevertheless, there is no effect on pre-CS freezing in the fear-conditioned without extinction groups between those treated with vehicle and those treated with VPA, indicating that context exposure alone is not responsible for this reduction of pre-CS freezing. Furthermore, our experiments testing the combination of VPA with partial extinction training indicate that cue exposure is crucial for any VPA effect on H4 acetylation.

The findings that fear conditioning with and without extinction result in distinct patterns of histone acetylation support, and extend to specific promoters of a single gene, the findings of Levenson et al. (2004), who show an increase in $\mathrm{H} 3$ acetylation in hippocampus after contextual fear conditioning and in $\mathrm{H} 4$ acetylation after latent inhibition, a form of inhibitory learning that shares common cellular substrates with extinction (Barad et al. 2005). However, our data suggest a more complicated story. Acetylated $\mathrm{H} 3$ appears to be increased by fear conditioning without extinction at both promoters, but it remains elevated around $\mathrm{P} 4$ after extinction, while extinction strongly depresses the amount of acetylated $\mathrm{H} 3$ associated with P1. H4 is little affected by fear conditioning without extinction at either promoter, and it remains largely unchanged by extinction training at P1. However, acetylated $\mathrm{H} 4$ associated with the P4 promoter is significantly increased by extinction training. Thus, in our experiments, different behavioral training protocols do not appear to affect any histone in general, but rather acetylation of specific combinations of histones associated with specific promoters. These data establish a rationale for future studies to examine specific components of the associative learning process (i.e., the effects of CS alone, or US alone, the contrast between unpaired and paired training, etc.) and to examine training effects on histone modification around the promoters of other candidate learning genes. Such studies will ultimately require increased specificity in the molecular analysis by examining specific populations of brain cells (i.e., glutamatergic or GABAergic neurons, or glia) before and after opposing forms of learning like the acquisition and extinction of conditioned fear.

Our results indicate that the HDAC inhibitors VPA and sodium butyrate facilitate long-term memory for extinction in a dose-dependent fashion, because of their HDAC-inhibiting activity (Fig. 4A,C). First, we see similar behavioral effects for two well-characterized HDAC inhibitors. Second, as a negative control we used valpromide, a compound that shares most of the structure and therapeutic effects of VPA, but is not an HDAC inhibitor. Valpromide had no effect on extinction learning, indicating that VPA potentiates extinction because of its HDACinhibitor, but not its anticonvulsant or mood-stabilizing effects (Fig. 4B). Indeed, previous work has demonstrated a role for HDAC inhibitors in enhancing long-term memory for conditioned fear with both systemic (Levenson et al. 2004) and local infusion (Yeh et al. 2004), as well as increasing long-term memory for spatial learning (Wood et al. 2005). An HDAC inhibitor can also reverse some of the effects of early life stress on fear-related behavior (Weaver et al. 2004).

To add to the evidence that VPA may exert its effects on extinction learning through changes in histone acetylation, we looked for further correlative evidence of the interaction between 


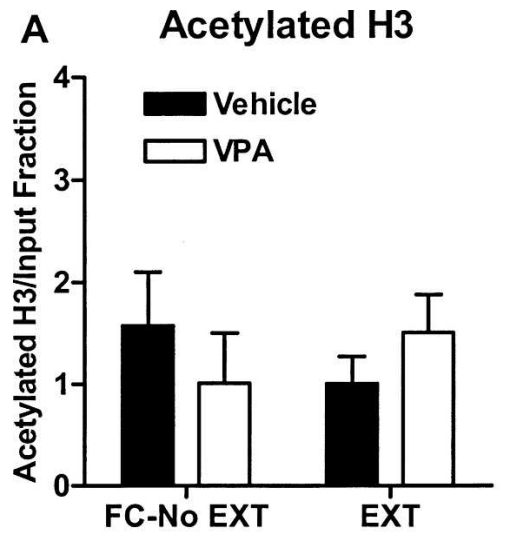

C

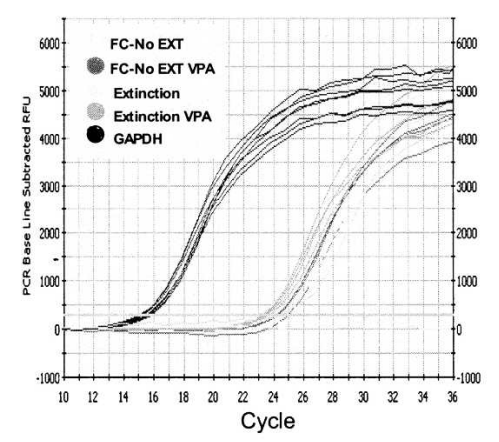

B $\quad$ Acetylated $\mathrm{H} 4$

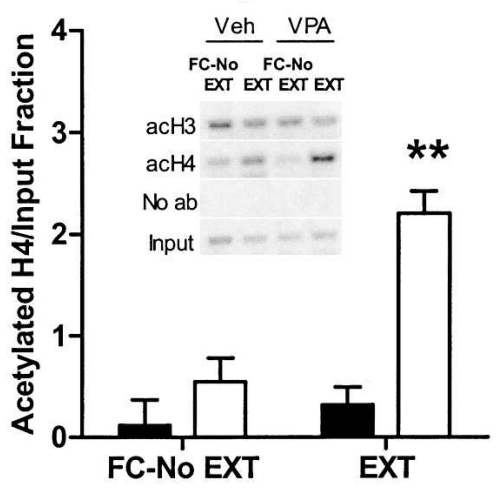

D BDNF Exon IV mRNA

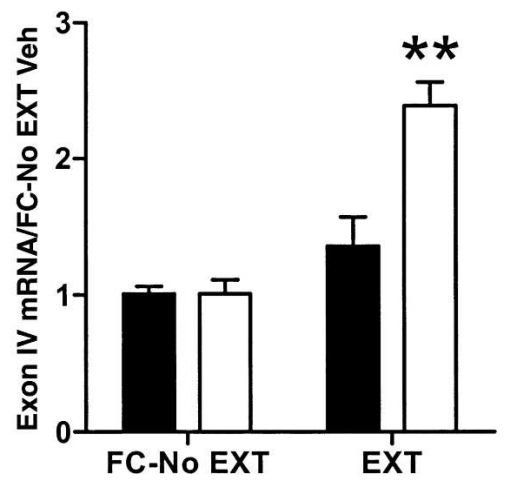

Figure 6. Effect of histone deacetylase inhibition by valproic acid (VPA) on learning-induced histone modifications $( \pm$ SEM) around the P4 promoter of the BDNF gene and on BDNF exon IV mRNA expression in the prefrontal cortex. VPA $(100 \mathrm{mg} / \mathrm{kg})$ combined with partial extinction training (20 CS presentations) $(A)$ had no effect on $\mathrm{H} 3$ acetylation around the P4 promoter of the BDNF gene, $(B)$ but increased histone $\mathrm{H} 4$ acetylation relative to all other treatment groups $\left({ }^{* *} P<0.01, n=4\right.$ samples pooled from two animals each). (Inset) Raw data. (C) Representative quantitative RT-PCR plot for the effect of VPA and partial extinction training on BDNF exon IV mRNA expression in the prefrontal cortex. (D) VPA (100 mg/kg) yielded no significant (ns) increase in exon IV mRNA expression in retention controls, but a significant increase with extinction. ( ${ }^{* *} P<0.01, n=6$ samples pooled from two animals each.) (FC-No EXT) Fear conditioned without extinction; (EXT) partial extinction.

VPA and extinction training at the level of histone acetylation associated with the two BDNF promoters we targeted in this study. Interestingly, while the combination of VPA and extinction caused a synergistic increase of $\mathrm{H} 4$ acetylation at both the P1 and $\mathrm{P} 4$ promoters, exon I-containing BDNF transcripts were increased as much by extinction alone as by the combination of VPA and extinction. Exon IV-containing transcripts, however, were only increased by the combination of VPA and partial extinction training. Thus, BDNF exon IV mRNA expression is preferentially sensitive to HDAC inhibition and appears to be associated with learning the tone-no shock association (as occurs during extinction). BDNF exon I mRNA expression appears to be more sensitive to CS-alone presentations, and insensitive to the extinction association. This idea is consistent with the observation that higher numbers of CS presentations in the overtraining protocol used to measure the direct effect of behavioral extinction training on histone acetylation and BDNF mRNA expression have a more dramatic effect on BDNF exon I transcripts (Fig. 2B) than fewer CS presentations in the partial extinction protocol (even when combined with VPA treatment; Fig. 5D). In both the overtraining and the partial extinction protocols, the expression of BDNF exon I mRNA is uncorrelated with acetylation patterns of $\mathrm{H} 3$ and $\mathrm{H} 4$ at the $\mathrm{P} 1$ promoter, suggesting that learninginduced changes in acetylation of histones at the P1 promoter are not mechanistically connected to expression of the BDNF exon I mRNA. In contrast, the increase in BDNF exon IV mRNA expression with extinction is not only correlated with extinction learning, but is similar is magnitude between extinction overtraining (Fig. 2D) and the combination of partial extinction and valproate treatment (Fig. 6D). The contrast in the correlation between extinction learning and the expression of BDNF transcripts containing the two different exons suggests three possibilities: (1) the BDNF transcripts containing different exons may have distinct functions within a single cell, (2) the differing BDNF transcripts may have the same molecular function but act in different compartments of the same cell, or (3) the differing BDNF transcripts have the same molecular function but act in different populations of cells. Our data do not allow us to distinguish between these possibilities, but we favor the third, as it would be consistent with a model in which different aspects of learning are subserved by different cell populations.

The effect of HDAC inhibition on fear extinction and on $\mathrm{H} 4$ acetylation around $\mathrm{P} 4$ appears to be contingent on behavioral training at the time of drug administration, since when HDAC inhibitors are injected into fear-conditioned animals that get no extinction training, there is no effect either on fear responding to the CS at test (Fig. 4), or on $\mathrm{H} 4$ acetylation (Figs. 5, 6). Thus even though HDAC inhibition by these compounds is likely to be both nonspecific and widespread, they can still have specific effects guided by behavioral training. In fact, while VPA blocks histone deacetylation, we see no effect of VPA on H3 acetylation at the P4 promoter (Fig. 6A), and even see decreases in $\mathrm{H} 3$ acetylation around the $\mathrm{P} 1$ promoter (Fig. 5A).

There are at least 11 known HDACs, each with some degree of regional specificity in the mammalian brain (Sugino et al. 2006). It would of great interest to determine whether specific HDACS show preference for gene promoters associated with $\mathrm{H} 3$ or H4, and/or whether there is also neuronal (or glial) subtype specificity further regulating their action. We hypothesize that regional and cell-type specificities of HDAC expression may play a critical role in translating different forms of learning into sustained changes of gene expression. In fact, experiments using viral vectors indicate that HDAC5 activity may play a role in conditioned defeat (Tsankova et al. 2006), and that some class IIa calcium-regulated HDACs facilitate the development of cocaine addiction (Kumar et al. 2005).

In conclusion, our data suggest a relationship between histone $\mathrm{H} 4$ modifications at BDNF promoters and long-term memory for extinction of conditioned fear. Histone modifications have been associated with models of neuropsychiatric disorders including schizophrenia, depression, and addiction (Kumar et al. 2005; Sharma 2005; Tsankova et al. 2006), supporting a potential role for epigenetic mechanisms in the regulation of a 
wide variety of behaviors. Our studies provide new information about the potential role of epigenetic regulation of gene expression in a preclinical model of anxiety disorders and their treatment. The facilitating effects of VPA on extinction are particularly intriguing, since the drug is already in use in psychiatric treatment and can be used immediately as an adjunct to behavior therapy for human anxiety disorders.

\section{Materials and Methods}

\section{Subjects}

Naive 10-12-wk-old C57BL/6 male mice (Taconic Farms, Oxnard, CA) were housed four per cage, maintained on a 12-h light/dark schedule, and allowed free access to food and water. All testing was conducted during the light phase in illuminated testing rooms following protocols approved by the Institutional Animal Care and Use Committee of the University of California, Los Angeles.

\section{Drugs}

Valproic acid (30-300 mg/kg, i.p; Sigma), valpromide (a generous gift from Katwijk Chemie, Amsterdam, Holland) is five times more potent than VPA but is not an HDAC inhibitor) (Nau and Loscher 1986), and sodium butyrate (100-1000 mg/kg, i.p.; Sigma), were dissolved in 0.1 M PBS with several drops of DMSO. Mice were injected with vehicle or drug $2 \mathrm{~h}$ before behavioral testing. Drug pre-treatment time was chosen based on previous reports that indicate peak histone acetylation time-course postinjection (Tremolizzo et al. 2005).

\section{Conditioning apparatus}

Two contexts (A and B), in separate rooms, were used for all behavioral fear testing. Shuttle box compartments (Med Associates) measuring $20.3 \times 15.9 \times 21.3 \mathrm{~cm}$ served as context $\mathrm{A}$, and conditioning boxes (Med Associates) measuring $30.5 \times 24.1 \times 21$ $\mathrm{cm}$ served as context $\mathrm{B}$. Both contexts had two transparent walls and stainless steel grid floors $(3.2 \mathrm{~mm}$ in diameter, $8 \mathrm{~mm}$ centers); however, the grid floors in context $\mathrm{B}$ were covered with flat white acrylic inserts to minimize context generalization. Context A was wiped down before testing with $10 \%$ ethanol, and context B was wiped down with $10 \%$ methanol. Individual videocameras were mounted in the ceiling of each chamber and connected via a quad processor to a standard videocassette recorder and monitor for videotaping and scoring of freezing. Grid floors were connected to a scrambled shock source (Med Associates). Auditory stimuli (Med Associates) were delivered via a speaker in the chamber wall. Delivery of stimuli was controlled with a personal computer and Med-PC software through a SmartCTL Interface System (DIG-716; Med Associates). Background white noise was maintained at $62 \mathrm{~dB}$ throughout behavioral testing.

\section{Behavioral protocols, effects of fear conditioning and extinction on histone acetylation, and BDNF mRNA expression}

Naive animals were not trained at all and remained in their home cages until sacrifice. For the other two groups, fear conditioning consisted of three pairings (2-min intertrial interval; ITI) of a 2-min, 80-dB, white noise conditioned stimulus (CS) coterminating with a 2-sec, $0.7-\mathrm{mA}$ footshock in context A. Mice were matched into equivalent treatment groups based on freezing during the third training CS. One day later, the conditioned mice were brought to context $\mathrm{B}$, where the extinction group was presented with 57 CS presentations (5-sec ITI) based on our previous work indicating that this number of CS exposures induces near complete extinction (Cain et al. 2005). The fear-conditioned without extinction group spent an equivalent amount of time in context B without any CS presentations. Tissue was collected from both of these groups $2 \mathrm{~h}$ after the end of their context $\mathrm{B}$ session.

\section{Behavioral protocols: Histone deacetylase inhibitor interactions with partial extinction of conditioned fear}

Experiments investigating the effects of HDAC inhibitors on the extinction of conditioned fear consisted of three phases: fear acquisition (context A), fear extinction (context B), and testing (context B). Testing occurred $7 \mathrm{~d}$ after extinction training to allow for complete memory consolidation. In all experiments, cue fear was induced in untreated, naive mice with three pairings of a 2-min, 80-dB, white noise CS coterminating with a 2-sec, 0.7$\mathrm{mA}$ footshock (2-min ITI). Mice were matched into equivalent treatment groups based on freezing during the third training CS. One day later, after injections, mice were placed in context $\mathrm{B}$ and allowed to acclimate for $2 \mathrm{~min}$. Partial extinction training comprised 20 non-reinforced 2-min CS presentations (5-sec ITI) and was chosen because it yields, by itself, no long-term memory for extinction, based on our previous work using partial extinction to test the ability of different compounds to facilitate extinction (Cain et al. 2003; Ponnusamy et al. 2005). As controls, fearconditioned mice without extinction were injected with vehicle or drug and placed in context B for a time equal to that spent there by extinguished mice but were not exposed to any CS presentations. For the behavioral tests in Figure 2, $7 \mathrm{~d}$ after extinction, all mice were returned to context B in the drug-free state. After a 2-min acclimation, freezing was assessed during two 2-min CS presentations (2-min ITI). For the measures of histone acetylation and of BDNF exon I-IV mRNA accumulation in Figures 4 and 5 , tissue was collected $2 \mathrm{~h}$ after partial extinction training.

\section{Data analyses}

Behavioral freezing, the absence of all non-respiratory movements, was rated during all phases by an experienced investigator blind to subject treatment, using a 5-sec instantaneous time sampling technique. The percentage of observations with freezing was calculated for each mouse, and data represent mean \pm SEM freezing percentages for groups of mice during specified time bins. Total session means were analyzed with one-way ANOVA for the data in Figures 2, and by two-way ANOVA for the data in Figures 3 and 5. Bonferroni post hoc tests were used for acetylation data and mRNA experiments; Dunnet's post hoc tests with vehicle extinction as reference were used for behavioral data (Fig. 3).

\section{Chromatin immunoprecipitation}

Chromatin immunoprecipitation (ChIP) was performed following a modification of the Upstate Biotechnology ChIP kit protocol. Tissue was fixed in 1\% formaldehyde. Cross-linked cell lysates were sheared by sonication in a $1 \%$ SDS lysis buffer to generate chromatin fragments with an average length of 100-200 bp. The chromatin was then immunoprecipitated using antibodies specific to acH3, which recognizes acetylated Lys9 and Lys14 (Upstate Biotechnology), or acH4, which recognizes acetylated Lys5, Lys8, Lys12, and Lys16 (Upstate Biotechnology), or an equivalent amount of control IgG (anti-rabbit; Santa Cruz) overnight at $4^{\circ} \mathrm{C}$. Protein-DNA-antibody complexes were precipitated with protein A-agarose beads coated with sheared salmon sperm DNA for $1 \mathrm{~h}$ at $4^{\circ} \mathrm{C}$, followed by two washes in low salt buffer, two washes in high salt buffer, two washes in LiCl buffer, and three washes with $1 \times$ TE. The precipitated protein-DNA complexes were eluted from the antibody with $1 \%$ SDS and $0.1 \mathrm{M}$ $\mathrm{NaHCO}_{3}$, then incubated overnight at $65^{\circ} \mathrm{C}$ in $200 \mathrm{mM} \mathrm{NaCl}$ to reverse formaldehyde cross-links. Following proteinase $\mathrm{K}$ digestion, phenol-chloroform extraction, and ethanol precipitation, samples were subjected to 32-35 cycles of PCR amplification using primer pairs specific for 200-bp segments corresponding to promoters upstream of mouse exons I and IV and $\beta$-tubulin (used previously as a negative control by Tsankova et al. 2004). Primer sequences are as follows: exon I: forward, 5'-TTCGATTCACG CAGTTGTTC-3', reverse, 5'-CTGAGCCAGTTACGTGACCA-3'; exon IV: forward, 5'-GCGCGGAATTCTGATTCTGG-3', reverse, 5'-AAAGTGGGTGGGAGTCCA-3'; $\beta$-tubulin: forward, 5 '-TA 
GAACCTTCCTGCGGTCGT-3'; reverse, 5'-TTTTCTTCTGGGCTG GTCTC-3'.

\section{Data analyses}

Histone acetylation levels were calculated from standard PCRamplified, ChIP products using a semiquantitative method, normalized to bands amplified from input DNA. Calculated histone acetylation levels were expressed as a ratio of that derived from naive animals (Fig. 2), and for Figures 4 and 5 data are presented normalized to bands amplified from input DNA. Mean histone acetylation levels for different groups were analyzed by one-way (Fig. 2) or two-way (Figs. 4, 5) ANOVA with Bonferroni post-tests.

\section{Quantitative RT-PCR}

RNA from the prefrontal cortex was prepared using the Trizol extraction method (Invitrogen). Total RNA ( $1 \mu \mathrm{g})$ was used for cDNA synthesis using the Omniscript RT kit (QIAGEN). PCR was then performed using a forward primer within BDNF exon I (5'AGTTGCTTTGTCTTCTGTAGTCGC-3') and a reverse primer (5'CCTGGAGACTCAGTGTCTTA-3'), and a forward primer for BDNF exon IV (5'-CAGGAGTACATATCGGCCACCA-3') and a reverse primer ( $5^{\prime}$-GTAGGCCAAGTTGCCTTGTCCGT-3'). Primers specific for GAPDH were used as a control (forward, 5'-GTCA TATTTCTCGTGGTTCACACC-3'; reverse, 5'-CTGAGTATGTCGT GGAGTCTACTGG-3'). Quantitative real-time PCR was performed in an iCycler (Bio-Rad) with the use of SYBR-green (PE Applied Biosystems). The threshold cycle for each sample was chosen from the linear range and converted to a starting quantity by interpolation from a standard curve run on the same plate for each set of primers. The BDNF exon I-IV mRNA levels were normalized for each well to the GAPDH mRNA levels using the $\Delta \Delta C T$ method, and each PCR reaction, run in duplicate for each sample, was repeated at least two independent times. (For color images of representative quantitative RT-PCR plots, please contact the corresponding author.)

\section{Data analysis}

Normalized exon I and IV mRNA levels were expressed as a ratio of those from retention control, vehicle-treated animals. mRNA levels were analyzed by two-way ANOVA followed by Bonferroni post-tests.

\section{Acknowledgments}

This work was supported in part by grants from the NIMH and the Tennenbaum Family Foundation (M.B.) and by postdoctoral fellowships from FRSQ and NSERC (T.W.B.).

\section{References}

Barad, M., Blouin, A.M., and Cain, C.K. 2005. Like extinction, latent inhibition of conditioned fear in mice is blocked by systemic inhibition of L-type voltage-gated calcium channels. Learn. Mem. 11: $536-539$

Baum, M. 1988. Spontaneous recovery from the effects of flooding (exposure) in animals. Behav. Res. Ther. 26: 185-186.

Berman, D.E. and Dudai, Y. 2001. Memory extinction, learning anew, and learning the new: Dissociations in the molecular machinery of learning in cortex. Science 291: 2417-2419.

Bouton, M.E. and King, D.A. 1983. Contextual control of the extinction of conditioned fear: Tests for the associative value of the context. $J$. Exp. Psychol. Anim. Behav. Process. 9: 248-265.

Cain, C.K., Blouin, A.M., and Barad, M. 2003. Temporally massed CS presentations generate more fear extinction than spaced presentations. J. Exp. Psychol. Anim. Behav. Process. 29: 323-333.

Cain, C.K., Godsil, B.P., Jami, S., and Barad, M. 2005. The L-type calcium channel blocker nifedipine impairs extinction, but not reduced contingency effects, in mice. Learn. Mem. 12: 277-284.

Cammarota, M., Bevilaqua, L.R., Kerr, D., Medina, J.H., and Izquierdo, I. 2003. Inhibition of mRNA and protein synthesis in the CA1 region of the dorsal hippocampus blocks reinstallment of an extinguished conditioned fear response. J. Neurosci. 23: 737-741.

Castro, L.M., Gallant, M., and Niles, L.P. 2005. Novel targets for valproic acid: Up-regulation of melatonin receptors and neurotrophic factors in C6 glioma cells. J. Neurochem. 95: 1227-1236.
Chhatwal, J.P., Stanek-Rattiner, L., Davis, M., and Ressler, K.J. 2006. Amygdala BDNF signaling is required for consolidation but not encoding of extinction. Nat. Neurosci. 9: 870-872.

Chwang, W.B., O'Riordan, K.J., Levenson, J.M., and Sweatt, J.D. 2006. ERK/MAPK regulates hippocampal histone phosphorylation following contextual fear conditioning. Learn. Mem. 13: 322-328.

Foley, A.G., Gallagher, H.C., Murphy, K.J., and Regan, C.M. 2004. Pentyl-4-yn-valproic acid reverses age-associated memory impairment in the Wistar rat. Neurobiol. Aging 25: 539-546.

Fukumoto, T., Morinobu, S., Okamoto, Y., Kagaya, A., and Yamawaki, S. 2001. Chronic lithium treatment increases the expression of brain-derived neurotrophic factor in the rat brain. Psychopharmacology 158: 100-106.

Herry, C. and Mons, N. 2004. Resistance to extinction is associated with impaired immediate early gene induction in medial prefrontal cortex and amygdala. Eur. J. Neurosci. 20: 781-790.

Herry, C., Trifilieff, P., Micheau, J., Luthi, A., and Mons, N. 2006. Extinction of auditory fear conditioning requires MAPK/ERK activation in the basolateral amygdala. Eur. J. Neurosci. 24: 261-269.

Kumar, A., Choi, K.H., Renthal, W., Tsankova, N.M., Theobald, D.E., Truong, H.T., Russo, S.J., Laplant, Q., Sasaki, T.S., Whistler, K.N., et al. 2005. Chromatin remodeling is a key mechanism underlying cocaine-induced plasticity in striatum. Neuron 48: 303-314.

Lattal, K.M., Radulovic, J., and Lukowiak, K. 2006. Extinction: Does it or doesn't it? The requirement of altered gene activity and new protein synthesis. Biol. Psychiatry 60: 344-351.

Levenson, J.M., O’Riordan, K.J., Brown, K.D., Trinh, M.A., Molfese, D.L., and Sweatt, J.D. 2004. Regulation of histone acetylation during memory formation in the hippocampus. J. Biol. Chem. 279: 40545-40559.

Levenson, J.M., Roth, T.L., Lubin, F.D., Miller, C.A., Huang, I.C., Desai, P., Malone, L.M., and Sweatt, J.D. 2006. Evidence that DNA (cytosine-5) methyltransferase regulates synaptic plasticity in the hippocampus. J. Biol. Chem. 281: 15763-15773.

Li, S., Murakami, Y., Wang, M., Maeda, K., and Matsumoto, K. 2006. The effects of chronic valproate and diazepam in a mouse model of posttraumatic stress disorder. Pharmacol. Biochem. Behav. 85: $324-331$.

Lin, C.H., Yeh, S.H., Lu, H.Y., and Gean, P.W. 2003. The similarities and diversities of signal pathways leading to consolidation of conditioning and consolidation of extinction of fear memory. $J$. Neurosci. 23: 8310-8317.

Martinowich, K., Hattori, D., Wu, H., Fouse, S., He, F., Hu, Y., Fan, G., and Sun, Y.E. 2003. DNA methylation-related chromatin remodeling in activity-dependent BDNF gene regulation. Science 302: 890-893.

Milad, M.R. and Quirk, G.J. 2002. Neurons in medial prefrontal cortex signal memory for fear extinction. Nature 420: 70-74.

Milad, M.R., Vidal-Gonzalez, I., and Quirk, G.J. 2004. Electrical stimulation of medial prefrontal cortex reduces conditioned fear in a temporally specific manner. Behav. Neurosci. 118: 389-394.

Murphy, K.J., Fox, G.B., Foley, A.G., Gallagher, H.C., O'Connell, A., Griffin, A.M., Nau, H., and Regan, C.M. 2001. Pentyl-4-yn-valproic acid enhances both spatial and avoidance learning, and attenuates age-related NCAM-mediated neuroplastic decline within the rat medial temporal lobe. J. Neurochem. 78: 704-714.

Nau, H. and Loscher, W. 1986. Pharmacologic evaluation of various metabolites and analogs of valproic acid: Teratogenic potencies in mice. Fundam. Appl. Toxicol. 6: 669-676.

Ou, L.C. and Gean, P.W. 2006. Regulation of amygdala-dependent learning by brain-derived neurotrophic factor is mediated by extracellular signal-regulated kinase and phosphatidylinositol-3-kinase. Neuropsychopharmacology 31: 287-296.

Phiel, C.J., Zhang, F., Huang, E.Y., Guenther, M.G., Lazar, M.A., and Klein, P.S. 2001. Histone deacetylase is a direct target of valproic acid, a potent anticonvulsant, mood stabilizer, and teratogen. J. Biol. Chem. 276: 36734-36741.

Ponnusamy, R., Nissim, H.A., and Barad, M. 2005. Systemic blockade of D2-like dopamine receptors facilitates extinction of conditioned fear in mice. Learn. Mem. 12: 399-406.

Rattiner, L.M., Davis, M., and Ressler, K.J. 2004. Differential regulation of brain-derived neurotrophic factor transcripts during the consolidation of fear learning. Learn. Mem. 11: 727-731.

Rescorla, R.A. and Heth, C.D. 1975. Reinstatement of fear to an extinguished conditioned stimulus. J. Exp. Psychol. Anim. Behav. Process. 1: 88-96.

Sangha, S., Scheibenstock, A., Morrow, R., and Lukowiak, K. 2003. Extinction requires new RNA and protein synthesis and the soma of the cell right pedal dorsal 1 in Lymnaea stagnalis. J. Neurosci. 23: 9842-9851.

Santini, E., Ge, H., Ren, K., Pena de Ortiz, S., and Quirk, G.J. 2004. Consolidation of fear extinction requires protein synthesis in the medial prefrontal cortex. J. Neurosci. 24: 5704-5710. 
Sharma, R.P. 2005. Schizophrenia, epigenetics and ligand-activated nuclear receptors: A framework for chromatin therapeutics. Schizophr. Res. 72: 79-90.

Sugino, K., Hempel, C.M., Miller, M.N., Hattox, A.M., Shapiro, P., Wu, C., Huang, Z.J., and Nelson, S.B. 2006. Molecular taxonomy of major neuronal classes in the adult mouse forebrain. Nat. Neurosci. 9: 99-107.

Suzuki, A., Josselyn, S.A., Frankland, P.W., Masushige, S., Silva, A.J., and Kida, S. 2004. Memory reconsolidation and extinction have distinct temporal and biochemical signatures. J. Neurosci. 24: 4787-4795.

Tremolizzo, L., Doueiri, M.S., Dong, E., Grayson, D.R., Davis, J., Pinna, G., Tueting, P., Rodriguez-Menendez, V., Costa, E., and Guidotti, A. 2005. Valproate corrects the schizophrenia-like epigenetic behavioral modifications induced by methionine in mice. Biol. Psychiatry 57: 500-509.

Tsankova, N.M., Kumar, A., and Nestler, E.J. 2004. Histone modifications at gene promoter regions in rat hippocampus after acute and chronic electroconvulsive seizures. J. Neurosci. 24: $5603-5610$.

Tsankova, N.M., Berton, O., Renthal, W., Kumar, A., Neve, R.L., and Nestler, E.J. 2006. Sustained hippocampal chromatin regulation in a mouse model of depression and antidepressant action. Nat. Neurosci. 9: 519-525.

Vianna, M.R., Igaz, L.M., Coitinho, A.S., Medina, J.H., and Izquierdo, I. 2003. Memory extinction requires gene expression in rat hippocampus. Neurobiol. Learn. Mem. 79: 199-203.
Weaver, I.C., Cervoni, N., Champagne, F.A., D'Alessio, A.C., Sharma, S., Seckl, J.R., Dymov, S., Szyf, M., and Meaney, M.J. 2004. Epigenetic programming by maternal behavior. Nat. Neurosci. 7: 847-854.

Winterer, G. and Hermann, W.M. 2000. Valproate and the symptomatic treatment of schizophrenia spectrum patients. Pharmacopsychiatry 33: $182-188$.

Wood, M.A., Vecsey, C., Lattal, K., Stein, J.M., Fabian, S.A., Attner, M.A. and Abel, T. 2005. Induced histone hyperacetylation enhances memory storage and facilitates synaptic plasticity via the transcription factor CREB. Abstract Viewer/Intinerary Planner Online. Society for Neuroscience, Washington, DC.

Wood, M.A., Hawk, J.D., and Abel, T. 2006. Combinatorial chromatin modifications and memory storage: A code for memory? Learn. Mem. 13: $241-244$.

Yang, Y.L. and Lu, K.T. 2005. Facilitation of conditioned fear extinction by $\mathrm{D}$-cycloserine is mediated by mitogen-activated protein kinase and phosphatidylinositol 3-kinase cascades and requires de novo protein synthesis in basolateral nucleus of amygdala. Neuroscience 134: 247-260.

Yeh, S.H., Lin, C.H., and Gean, P.W. 2004. Acetylation of nuclear factor- $\kappa \mathrm{B}$ in rat amygdala improves long-term but not short-term retention of fear memory. Mol. Pharmacol. 65: 1286-1292.

Received December 8, 2006; accepted in revised form February 20, 2007.

\section{Learning \& Memory}




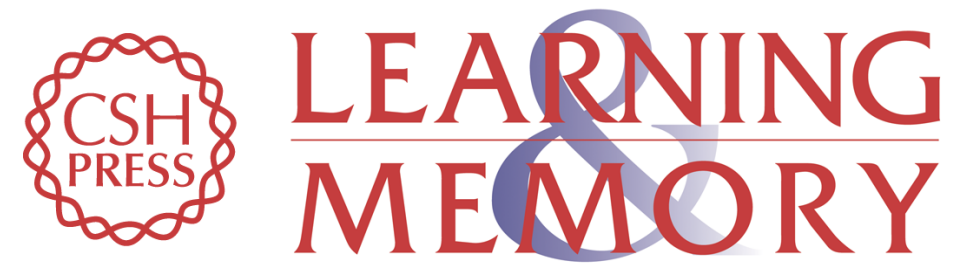

\section{Histone modifications around individual BDNF gene promoters in prefrontal cortex are associated with extinction of conditioned fear}

Timothy W. Bredy, Hao Wu, Cortney Crego, et al.

Learn. Mem. 2007, 14:

Access the most recent version at doi:10.1101//m.500907

References This article cites 44 articles, 17 of which can be accessed free at:

http://learnmem.cshlp.org/content/14/4/268.full.html\#ref-list-1

License

Email Alerting Receive free email alerts when new articles cite this article - sign up in the box at the Service top right corner of the article or click here. 